\title{
Atomic Resolution Imaging of Metal Oxide Nanoparticles
}

Y.-M. Kim, ${ }^{*}$ H.J. Kim, ${ }^{* *}$ J.Y. Lee, ${ }^{* * *}$ Y.-J. Kim ${ }^{*}$

*Division of Electron Microscopic Research and ${ }^{* *}$ Division of Materials Science, Korea Basic Science Institute, Daejeon 305-334, Korea

${ }^{* * *}$ Department of Materials Science and Engineering, KAIST, Daejeon 305-701, Korea

Seeing oxygen atoms has been of great interest because the oxygen occupancy of certain lattice sites sensitively controls the materials properties [1]. Nowadays, seeing light elements in materials is becoming a routine of materials scientists with the help of aberration-corrected TEM/STEMs [2,3]. However, atomic-scale analysis of metal oxide nanoparticles with the oxygen configuration resolved remains challenging due to practical hindrances to microscopic imaging. To image light elements inside nanoparticles has two huddles more, that is, interferences of support films and confined thicknesses of nanoparticles: Contrast interference of amorphous support film strongly affects image intensity for the nanoparticle, which sometime spoils accurate interpretation for the atomic structure even with the use of state-of-the-art medium-voltage TEMs. Confined sizes of nanoparticles generally raise a fundamental issue because nanoparticles of interest often stand off the thickness range possible for directly interpretable atomic column imaging condition. In these respects, high energy electrons in high voltage electron microscope (HVEM operated at $1250 \mathrm{keV}$ ) can mitigate these difficulties to image an atomic structure of oxide nanoparticle supported by an amorphous film: The high transmission power of the HVEM reduces the interference of background contrast and simultaneously provides relatively wide range of thickness for directly interpretable imaging condition.

In this paper, we report direct atomic-scale imaging of magnetic $\mathrm{NiO}$ nanoparticles with the information of oxygen configuration inside the particle. Furthermore, the microscope conditions to obtain oxygen contrast and the relevant quantification will be discussed combined with highresolution image calculations of $\mathrm{NiO}$ structure (see Fig. 1 and 2). Seeing oxygen in magnetic nanoparticles becomes now possible by applying HVEM technique with a full atomic resolution. Since the oxygen is a key element for material properties in ceramic nanocompounds, this result means we have one more powerful tool for understanding how to deal with such nanomaterials.

\section{References}

[1] J.C.H. Spence, Science 299 (2003) 839.

[2] C.L. Jia et al., Science 303 (2004) 2001.

[3] O.L. Krivanek et al., Nature 464 (2010) 571.

[4] This research was supported by KBSI grant (T3021B). 

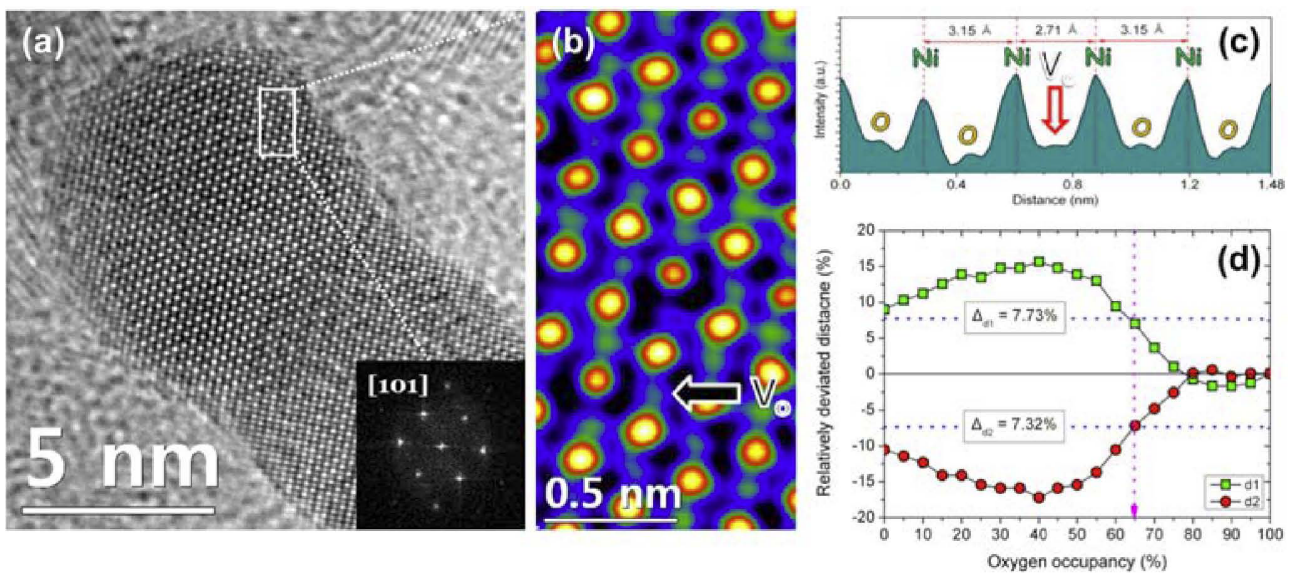

FIG. 1. (a) Experimental high-resolution image of $\mathrm{NiO}$ [101] nanoparticle. (inset: diffractogram of a). (b) The enlarged image of the region-of-interest marked as rectangular in $\mathbf{a}$. The solid arrow indicates the position of oxygen deficient column in the image, which was identified by tracing the relative deviation from the reference nickel-nickel atomic distance $(2.92 \AA)$ as a function of oxygen occupancy (see graph d). Note the small deviations from the regular $\mathrm{Ni}-\mathrm{Ni}$ atomic distance is attributed from nonlinear contrast contribution and does not reflect the real deviation. (c) The intensity profile of the atomic columns including the oxygen deficient site marked as arrow. (d) Estimating the possible oxygen occupancy of the identified oxygen deficient column as shown in $\mathbf{b}$ and $\mathbf{c}$.

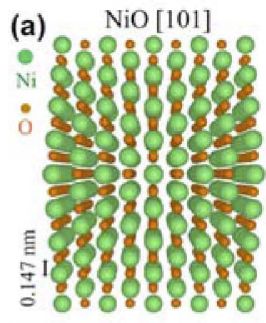

(d)

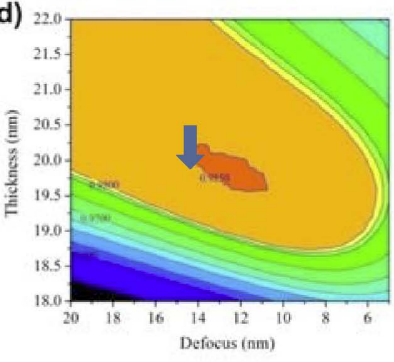

FIG. 2. (a) Atomic structure model of $\mathrm{NiO}$ [101] nanoparticle. Note the noise was filtered by Wiener filtering method. (c) The calculated image of NiO [101] at the imaging condition of thickness, $t=20 \mathrm{~nm}$ and defocus, $f=+12 \mathrm{~nm}$, resulting in the best cross-correlation $(\mathrm{XCF}=0.99)$. (d) Statistical cross-correlation map between the experimental image and the calculated images as functions of isotropic imaging parameters, thickness and defocus. (e) Contrast transfer function (CTF) at the experimental imaging condition. 